\title{
Digital Imaging Systems for Plain Radiography
}

Lança, Luis, Silva, Augusto

2013, XVI, 115 p. 84 illus., 46 in color.

Available Formats:

eBook

$99,99 €$

(gross) price

ISBN 978-1-4614-5067-2

Immediately available per PDF-download (no DRM, watermarked)

Hardcover

Information

$106,95 €$

Advances in digital technology led to the development of digital x-ray detectors that are currently in wide use for projection radiography, including Computed Radiography (CR) and Digital Radiography (DR). Digital Imaging Systems for Plain Radiography addresses the current technological methods available to medical imaging professionals to ensure the optimization of the radiological process concerning image quality and reduction of patient exposure. Based on extensive research by the authors and reference to the current literature, the book addresses how exposure parameters influence the diagnostic quality in digital systems, what the current acceptable radiation doses are for useful diagnostic images, and at what level the dose could be reduced to maintain an accurate diagnosis. The book is a valuable resource for both students learning the field and for imaging professionals to apply to their own practice while performing radiological examinations with digital systems

Content Level » Professional/practitioner

Keywords » PACS - digital radiography - image quality - informatics - patient dose

Related subjects » Public Health - Radiology

\section{TABLE OF CONTENTS}

Introduction.- Digital radiography detectors: a technical overview.- Digital radiography detector performance.- Technical considerations concerning digital technologies.- Assessment of patient dose in digital systems.- Image quality in diagnostic radiology.- Practical insights into digital radiology.- Image enhancement for digital radiography.- Digital radiography and Picture Archiving and Communication Systems (PACS). 